Annuaire suisse de politique de développement

26-2 | 2007

Financer le développement par la mobilisation des ressources locales

\title{
Pétrole et industries extractives, la société civile africaine se mobilise
}

\section{Catherine Morand}

\section{CpenEdition}

\section{Journals}

Édition électronique

URL : http://journals.openedition.org/aspd/125

DOI : 10.4000/aspd. 125

ISSN : 1663-9669

Éditeur

Institut de hautes études internationales et du développement

Édition imprimée

Date de publication : 1 novembre 2007

Pagination : 99-101

ISBN : 978-2-88247-068-3

ISSN : 1660-5934

Référence électronique

Catherine Morand, « Pétrole et industries extractives, la société civile africaine se mobilise », Annuaire suisse de politique de développement [En ligne], 26-2 | 2007, mis en ligne le 19 juin 2009, consulté le 07 septembre 2020. URL : http://journals.openedition.org/aspd/125 ; DOI : https://doi.org/10.4000/aspd. 125 


\title{
Pétrole et industries extractives, la société civile africaine se mobilise
}

\author{
Catherine Morand*
}

a société civile d'un nombre croissant de pays africains riches en pétrole, mais aussi en minerais, se mobilise avec un courage exemplaire pour exiger une véritable transparence aussi bien de leurs gouvernements que des compagnies actives dans ce secteur, afin que les populations des pays concernés puissent voir leurs conditions de vie s'améliorer. C'est ce qu'a pu constater l'auteure en mars 2007 au Cameroun, lors d'un meeting réunissant des représentants de l'ensemble des pays producteurs de pétrole et de minerais.

Au cours d'une conférence de presse qui s'est tenue le 26 avril 2007 à Abidjan, Michel Yoboué, responsable de la coalition «Publiez ce que vous payez" en Côte d'Ivoire, a annoncé le lancement d'une campagne nationale de sensibilisation afin «de demander des comptes sur I'utilisation des revenus pétroliers", tout en relevant le manque total de transparence quant à l'utilisation de la manne pétrolière qui prévaut en Côte d'Ivoire, "un pays qui n'est toujours pas classé comme pays producteur de pétrole alors qu'il en produit 90000 barils par jour", le double du Cameroun, pourtant indexé par le FMI parmi les pays producteurs de pétrole. "Nous souhaitons que la société civile joue sa partition dans la recherche de transparence dans la gestion du secteur pétrolier $"$, a encore insisté Michel Yoboué.

\section{Une véritable révolution}

Dans le contexte très opaque qui sévit dans le secteur pétrolier et minier en Côte d'Ivoire comme ailleurs sur le continent africain, il s'agit là d'une véritable révolution. Le fait qu'un nombre croissant d'initiatives visent à promouvoir de nouvelles règles du jeu permettant aux populations des pays producteurs de pétrole ou de minerais de bénéficier d'une gestion transparente et de voir leurs conditions de vie s'amélio- rer est particulièrement bienvenu. Car I'Afrique subsaharienne connaît actuellement, dans ces secteurs, la plus grande vague d'investissements que ce continent ait connus et une manne financière qui dépasse de loin l'aide au développement et financière octroyée à cette région. But de ces initiatives: parvenir à conjurer la «malédiction» de l'or noir, qui a surtout apporté aux pays africains producteurs de pétrole et de minerais catastrophes écologiques, corruption et maintien au pouvoir de régimes autoritaires.

Michel Yoboué faisait partie des nombreux représentants d'organisations de la société civile africaine membres de la campagne internationale "Publiez ce que vous payez" (PCOVP - en anglais: Publish What You Pay, PWYP) qui se sont retrouvés en mars 2007 à Limbe, au Cameroun, pour mettre au point une stratégie commune et acquérir les connaissances techniques nécessaires à ce combat.

J'y représentais Swissaid, Fondation suisse pour la coopération au développement, qui est très engagée sur ce thème par le biais de ses partenaires tchadiens, mais aussi nigériens et bissau-guinéens. C'est en apportant son appui à des associations tchadiennes luttant pour que les revenus pétroliers servent réellement à améliorer les conditions de vie de la population

* Journaliste, responsable de l'Antenne romande de Swissaid, Lausanne.

Cet article est un témoignage de la mobilisation des populations africaines dans le cadre de I'Initiative pour la transparence des industries extractives (EITI), traitée par Gilles Carbonnier dans sa contribution au présent dossier. 
que Swissaid a fait son entrée dans le monde "fascinant» du pétrole. Depuis le début des années 2000, Swissaid a donc accompagné la prise de parole de la société civile tchadienne et fait désormais de même au Niger et en Guinée-Bissau, deux pays où des prospections pétrolières sont à l'ordre du jour. Que ce soit dans le cadre d'un voyage de parlementaires et de journalistes suisses au Tchad en avril 2004 ou lors de plusieurs conférences organisées en Suisse, mais aussi au Niger et en Guinée-Bissau, Swissaid a toujours veillé à réunir l'ensemble des parties concernées par la question d'une gestion transparente des revenus pétroliers. Ce fut à nouveau le cas en novembre 2006 à Genève, où Swissaid a convié des représentants de sociétés pétrolières, d'organisations internationales, d'Etats producteurs de pétrole, de la société civile et d'organisations non gouvernementales (ONG) pour plancher sur le fait qu'en Afrique, "le pétrole n'est pas fatalement une fatalité».

Les pays africains jouent d'ailleurs un rôle de poids au sein de cette campagne à laquelle se sont associées de nombreuses organisations, actives dans les domaines de l'environnement ainsi que de la défense des droits humains et des droits syndicaux, et qui ont toutes un point commun: la lutte pour une gestion transparente des revenus des industries extractives. "Le fait de savoir combien d'argent est reçu par les gouvernements et combien est versé par les sociétés est une première démarche importante si I'on veut pouvoir demander des comptes et rendre responsables de ces sommes les autorités chargées de leur gestion", estime Matteo Pellegrini, coordinateur pour l'Afrique de la campagne PCQVP, lancée en 2002 et appuyée aujourd'hui par plus de 300 ONG dans le monde entier, qui pilotait cet atelier de formation.

\section{L'émergence de contre-pouvoirs}

Ces coalitions font aujourd'hui pression sur leurs gouvernements respectifs pour qu'ils rejoignent I'Initiative pour la transparence des industries extractives (en anglais: Extractive Industries Transparency Initiative, EITI), lancée par Tony Blair à I'occasion du Sommet mondial sur le développement durable à Johannesburg en
2002, qui mise sur une concertation entre représentants d'Etats, de sociétés pétrolières, d'organisations internationales et de la société civile en vue d'accroître la transparence des revenus générés par les compagnies pétrolières, gazières et minières. En filigrane: la conviction qu'une telle démarche est dans l'intérêt bien compris de I'ensemble des parties concernées, et la tentative d'éviter la multiplication des scénarios "à la nigériane", avec enlèvements et actes de sabotage, sur fond de misère quasi généralisée.

Les Tchadiens étaient venus en nombre au meeting de Limbe. C'est que la mobilisation de la société civile tchadienne, depuis qu'il avait été question de démarrer l'exploitation du pétrole dans ce pays, a largement contribué à l'émergence de contre-pouvoirs, et demeure une référence jusqu'à aujourd'hui. Dans un premier temps, la Banque mondiale avait apporté son concours et pesé de tout son poids pour favoriser une gestion transparente des revenus pétroliers, garantie par une loi, la Loi 001, unique en son genre, ensuite vidée de son contenu et remplacée par une Loi 002 faisant notamment l'impasse sur le Fonds pour les générations futures. Mais en ne parvenant pas à faire respecter par les autorités tchadiennes l'accord qui avait été conclu, l'institution financière s'est complètement décrédibilisée aux yeux des représentants de la société civile tchadienne et n'a désormais plus les moyens d'exercer une quelconque pression pour exiger une gestion transparente des revenus pétroliers de la part des dirigeants tchadiens. "Nous comptons désormais sur les pressions exercées dans le cadre de la campagne internationale PCQVP pour servir de levier auprès des autorités tchadiennes afin qu'elles rejoignent I'EITI», dit Gilbert Maoundonodji, responsable du GRAMP/TC, une ONG tchadienne appuyée par Swissaid et qui a rejoint cette campagne, à l'instar de la plupart des autres organisations de la société civile tchadienne mobilisées en faveur d'une gestion transparente des revenus pétroliers.

\section{L'expertise de la société civile tchadienne}

L'expertise de la société civile tchadienne, qui avait une longueur d'avance en matière de mobilisation dans un tel contexte, s'exporte 
désormais ailleurs sur le continent. C'est ainsi que Gilbert Maoundonodji, qui représente désormais l'Afrique au sein du Conseil international de I'EITI, dont le siège est à Oslo, a personnellement participé au lancement de l'initiative au Congo-Brazzaville et en Mauritanie. "L'expertise de la société civile tchadienne joue aujourd'hui un rôle moteur dans la campagne panafricaine de PCQVP», relève-t-il. L'atelier de formation de Limbe avait également pour but d'améliorer les compétences des représentants de la société civile pour qu'ils puissent être en mesure de jouer pleinement leur rôle dans ces divers processus, par exemple: savoir lire, comprendre et décrypter le budget de l'Etat et les contrats passés avec les sociétés pétrolières ou minières, maîtriser tous les aspects de l'EITI, les diverses législations, etc. "Nous devrions à terme pouvoir renégocier certains contrats, car les taxes qui sont reversées à nos Etats par les sociétés actives dans les industries extractives sont souvent dérisoires", déplore ainsi Steve Emmanuel Manteaw, de I'Integrated Social Development Centre au Ghana, une association qui travaille sur les impacts environnementaux et sociaux de l'exploitation minière dans ce pays.

En République démocratique du Congo, I'Initiative pour la transparence des industries extractives est aussi venue renforcer la courageuse mobilisation d'organisations de la société civile travaillant dans des conditions extrêmement difficiles. "Notre mobilisation sur I'exploitation des ressources naturelles du pays a commencé en 2000, raconte Jean-Pierre Muteba, responsable d'une organisation syndicale à Lubumbashi. L'EITI est arrivée bien à propos et nous permet notamment de dénoncer un Code minier adopté à la hâte, sous la pression de la Banque mondiale et dans une période de transition politique, qui ne respecte en rien les intérêts de notre pays. " Pour empêcher le bradage des richesses du sous-sol du Congo et obtenir de meilleures conditions de travail, il a lancé plusieurs mouvements de grève dans le secteur minier de la très riche province du Katanga, défiant ainsi de facto des autorités locales corrompues et la prédation des innombrables entreprises minières qui se ruent sur les mines de cuivre, de diamants, de coltane, de cobalt dont regorge la région, en la quasi-absence de tout contrôle étatique. II est régulièrement harcelé par des militaires et c'est une ONG hollandaise qui paie désormais des gardes armés pour veiller sur sa demeure et lui garantir un minimum de sécurité, à lui et à sa famille. "Nous devons nous battre, pour nous et nos enfants, pour empêcher le pillage de notre sous-sol, car nous n'avons nulle part d'autre où aller», explique encore Jean-Pierre Muteba.

\section{Harcèlement, pressions, arrestations...}

C'est que dans le secteur très sensible du pétrole et des ressources minières sur le continent africain, les personnes qui luttent pour une plus grande transparence de la gestion de ces revenus sont régulièrement l'objet de pressions, de menaces, voire d'arrestations. Les pressions émanent essentiellement des pouvoirs en place, mais aussi des entreprises. "Ce n'est pas parce que nous sommes régulièrement harcelés que nous allons renoncer à nous battre», estime toutefois Samuel Nsikabaka, professeur d'université à Brazzaville. Son pays résume à lui seul tout le drame de ce qu'il est convenu d'appeler la «malédiction du pétrole», ce paradoxe qui veut que plus un pays est riche en ressources naturelles, plus sa population croupit dans une scandaleuse pauvreté. II a profité des élections législatives en juin 2007 au Congo-Brazzaville pour interpeller les candidats et leur demander des comptes sur la gestion des fonds pétroliers. "Nous voulons que I'Etat nous dise comment ces fonds sont dépensés", insiste-t-il. "I a préféré mettre sa famille à l'abri en France, après que leur demeure eut été incendiée par des «inconnus». Deux membres de la coalition "Publiez ce que vous payez» du Congo-Brazzaville, Christian Mounzéo et Brice Mackosso, continuent à être assignés à résidence après avoir été arrêtés, détenus arbitrairement et condamnés pour diffamation à l'égard du chef de l'Etat. 